\title{
Imaging Findings of an Aggressive Multicompartmental Venous Vascular Malformation of the Skull Base
}

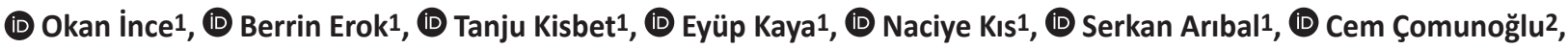 \\ (D) Hakan Önder 1
}

1 University of Health Sciences Turkey, Prof. Dr. Cemil Taşçığlu City Hospital, Clinic of Radiology, Istanbul, Turkey

2University of Health Sciences Turkey, Prof. Dr. Cemil Taşçığlu City Hospital, Clinic of Pathology, İstanbul, Turkey

\section{Abstract}

Primary vascular lesions are classified as vascular neoplasms and vascular malformations (VMs). Venous VM is the most frequent type of VMs with a prevalence of approximately $1 \%$ and is the most common venous anomaly in the head and neck region after hemangioma, but is rarely encountered in the skull base. An expansile growth pattern is noted with permeation into the tissue spaces by adjacent structure invasion, thus presenting with multicompartmental occupation, as in our case. Presented herein is a case of a 12 -year-old female patient complaining of left orbital swelling with an aggressive venous VM of the skull base predominantly occupying the left infratemporal fossa extending into various other compartments.

Keywords: Venous vascular malformation, cavernous hemangioma, bright T2W signal, infratemporal fossa, skull base imaging

\section{INTRODUCTION}

Primary vascular anomalies were simply categorized into hemangiomas and vascular malformations (VMs) by Mulliken and Glowacki (1) in 1982. Adopting this system based on the histological endothelial characteristics, the International Society for the Study of Vascular Anomalies classified the primary vascular lesions as vascular neoplasms and VMs in 1996 (2). VMs are subclassified into high (fast) flow VMs with arterial components (arterial malformation, arteriovenous malformation, and arteriovenous fistula), low (slow) flow VMs (lymphatic, venous, and capillary), and combined VMs (3). The main difference between vascular neoplasms is their non-proliferative nature despite growing in size with age, as well as non-involution with time in contrast to some vascular neoplasms, such as infantile hemangiomas (4). The previously named cavernous hemangiomas occur anywhere including the cerebral, hepatic, or vertebral cavernous hemangiomas instead of low flow venous VMs. A predilection for the oral cavity, airway, and muscle groups is found in the head and neck region (5). Infratemporal fossa (ITF) is an extremely rare location reported in a few cases in the literature (6).

Accurate preoperative diagnosis is very important to avoid operative complications. Reported are the imaging findings of an aggressive venous VM occupying several skull base compartments but primarily involving the left ITF in a 12-yearold female patient.

\section{CASE PRESENTATION}

A 12-year-old female patient presented with left orbital swelling. The head computed tomography (CT) revealed a left ITF mass (Figure 1). The magnetic resonance imaging (MRI) with gadolinium revealed an ITF mass with intermediate signal intensity on T1weighted (T1W) and bright signal intensity on T2W images, which was not attenuated on fluid-attenuated inversion recovery. The 
lesion extended into the pterygopalatine fossa, sphenoid sinus, posterior nasal cavity, and left cavernous sinus, where it partially surrounded the cavernous internal carotid artery, compressing the left maxillary sinus without invasion. However, by the left lateral orbital wall infiltration, it extended into the extraconal space, displacing the myofascial cone to the right in addition to the left pterygoid process and clival part of the sphenoid bone infiltration (Figure 2). Linear branching signal void areas were observed on the MRI, mimicking flow voids at first appearance. However, most of them were not enhanced on postcontrast images despite the prominent enhancement in the remaining part of the lesion (Figure 2). When simultaneously observed with the previous $\mathrm{CT}$, they correlated with the network of branching calcifications, which are evaluated as widespread phleboliths (Figure 1). No extension was found through the middle cranial fossa but some vasogenic edema in the adjacent temporal lobe without any pathological enhancement, probably due to

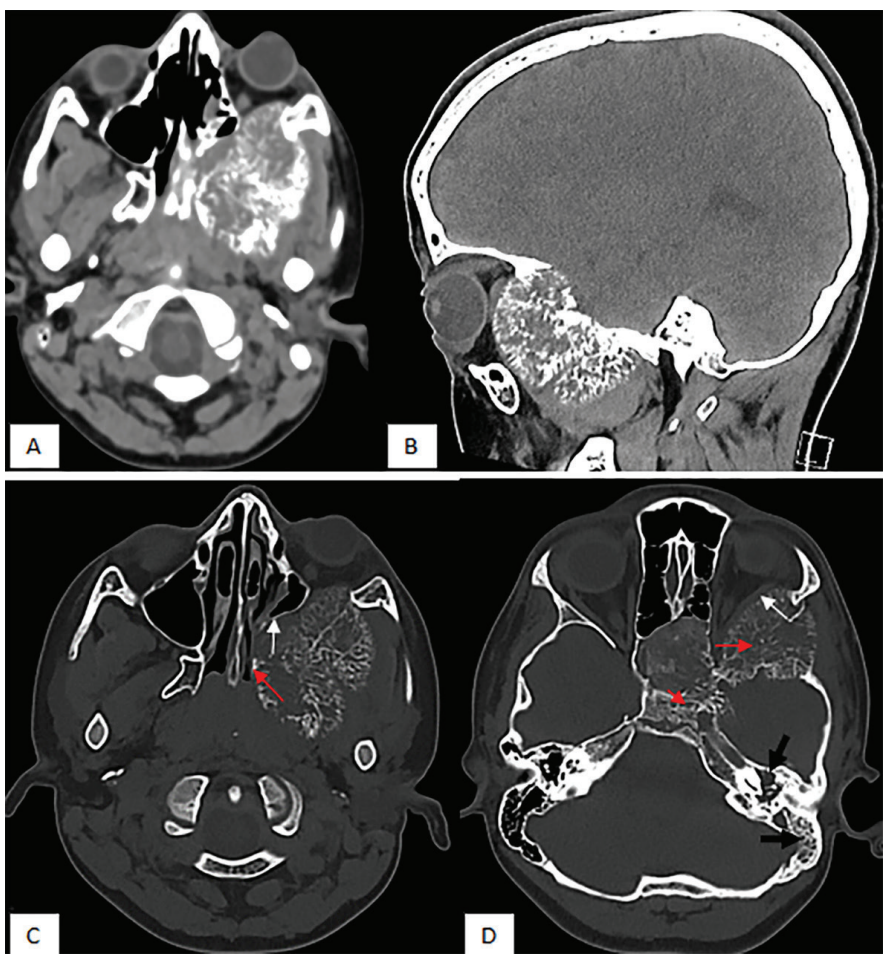

Figure 1. Head CT scan. Axial (A), sagittal (B) images at the intermediate window, and axial (C, D) images at the bone window show the large skull base lesion primarily located in the infratemporal fossa. The network of branching reticular calcifications (phleboliths) is visible throughout the lesion. Note the compression of the left maxillary sinus by the lesion $(C$; white arrow) and extraconal extension of the lesion with the myofascial cone displacement to the right (D, white arrow). Infiltration of the left lateral orbital wall (D, red arrow), the left pterygoid process (C, red arrow), and clival part of the sphenoid bone (D, short red arrow) are shown. Opacification of the tympanic cavity and mastoid air cells are also marked (D, black arrows)

CT: Computed tomography lesion compression (Figure 2). Venous VM was considered and flow dynamics were evaluated by external carotid artery digital subtraction angiography. Areas of blush-style enhancement from the ophthalmic and maxillary arteries, which were evident in the late venous phases and undetected in the early arterial phase, consistent with low flow VMs, were demonstrated (Figure 3). Surgical treatment was planned following endovascular sclerotherapy with orbital decompression as the first operation. The histopathological examination concluded the venous VM diagnosis (Figure 4).

\section{DISCUSSION}

Venous VM is the most frequent type of VMs with a prevalence of approximately $1 \%$ (7) and is the most common venous anomaly in the head and neck region after hemangiomas, but is rarely encountered in the skull base (2). Clinical presentation varies with the involved site. The ITF is a complex space, thus lesions are not realized for a long time until the compressive signs and symptoms associated with the adjacent structures occur, as in our case. In most cases, venous VMs are sporadic, but familial inheritance presented an association with blue rubber bleb nevus, Proteus ve Mafucci syndromes (8). Histologically, VMs are characterized with malformed, ectatic venous channels (phlebiectasis) with a slow stagnant flow that predisposes thrombosis and eventually phleboliths formation, which is the characteristic imaging feature of venous VMs (9). An expansile growth pattern with permeation into the tissue spaces by invading the adjacent structures is observed. The mural muscular anomalies cause the expansile growth pattern $(10,11)$. The radiological examination revealed a frequent finding of phleboliths, which are demonstrated on CT images as calcified linear hyperdensities. However, venous VM in the ITF, which was diagnosed by CT-guided biopsy in a 77-yearold female patient, was reported without this characteristic imaging feature (6), which was due to the early diagnosis with the patient complaining of maxillary sinusitis when the lesion was still small in size. MRI accurately shows the lesion extensions with their superior soft-tissue resolution. Owing to the low cellularity, venous VMs show characteristic bright T2W signals. Prominent contrast enhancement particularly at the late phases (90 s) is also a characteristic. The phleboliths are hypointense on MRI, which are confused with flow voids. However, flow voids are expected to be enhanced with contrast administration, whereas phleboliths are not. In addition, phleboliths show blooming artifacts on gradient-echo sequences. In our patient, the differential diagnosis includes proliferating hemangiomas, which are also hyperintense to the muscle on T2W images but not as bright as venous VMs (12). Juvenile nasopharyngeal 

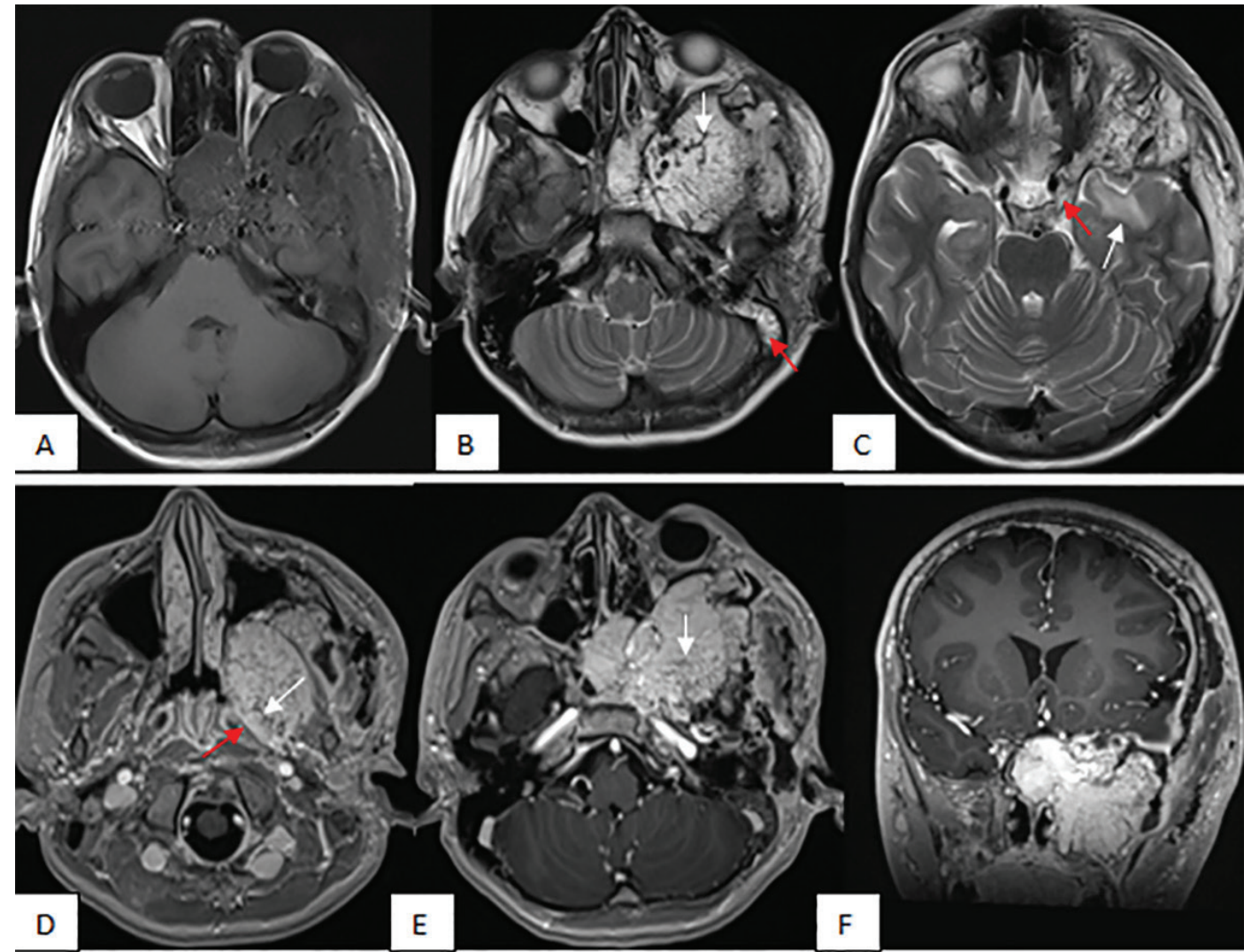

Figure 2: The brain MRI revealed a giant lesion located in its greatest part at the left infratemporal fossa having intermediate signal intensity on axial T1W (A) and bright signal intensity on axial T2W images (B, C) with extensions into the pterygopalatine fossa, sphenoid sinus, and posterior nasal cavity. Note the presence of signal void areas, some of which are in linear branching patterns (B; white arrow). Postcontrast axial (D, E) and coronal (F) images show prominent enhancement of the lesion except for most of the signal void areas (phleboliths) (D, E; white arrows). Note the extension into the left cavernous sinus where the cavernous ICA is partially surrounded (C; red arrow). The retropharyngeal space compression where the cartilaginous portion of the eustachian tube is affected (D; red arrow) resulting in secretory otomastoiditis (B, red arrow) is shown. Note also the edema in the adjacent temporal lobe ( $\mathrm{C}$, white arrow). The prominent trans-spatial occupation of the lesion is well demonstrated on the coronal contrast-enhanced T1W image $(F)$

MRI: Magnetic resonance imaging, T1W: T1-weighted, ICA: Internal carotid artery

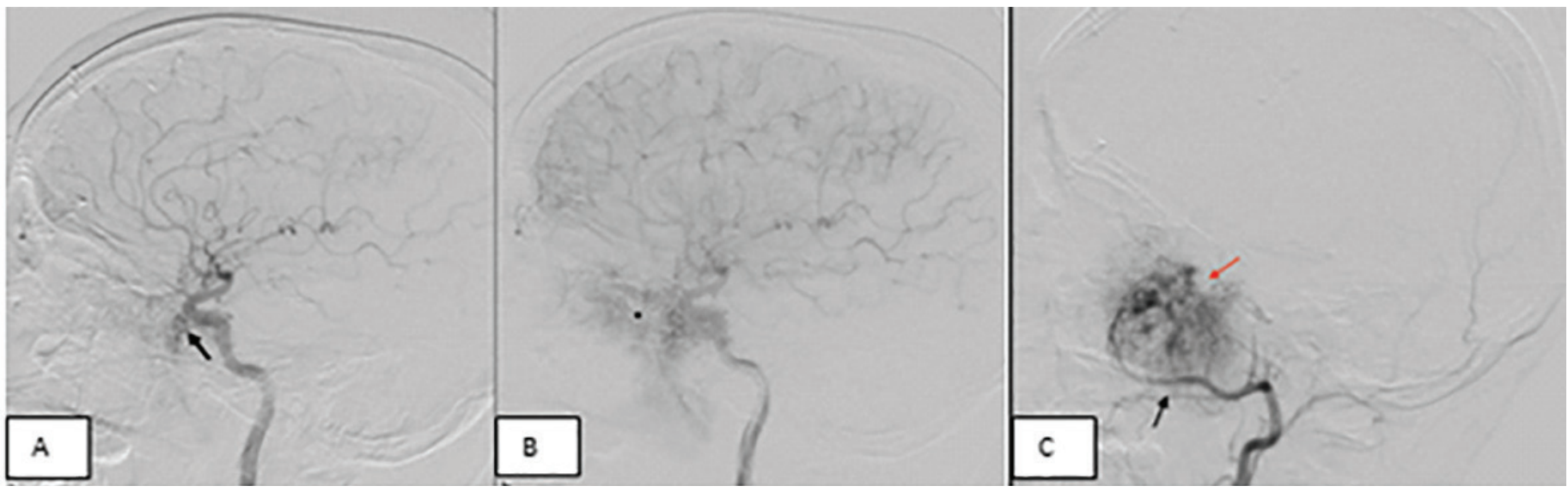

Figure 3: (A, B) DSA of the left ICA in lateral view: A) early arterial and B) late arterial images show the lesion supplied by ophthalmic artery of the ICA (A; arrow) and blush-like enhancement in the late arterial phase (B; asterisk). (C) DSA of the left ECA in lateral view shows the significant feeding of the lesion from the maxillary artery (black arrow) and blush-like enhancement (red arrow). Note the absence of tortuosity in the arterial and venous structures

DSA: Digital subtraction angiography, ICA: Internal carotid artery, ECA: External carotid artery 


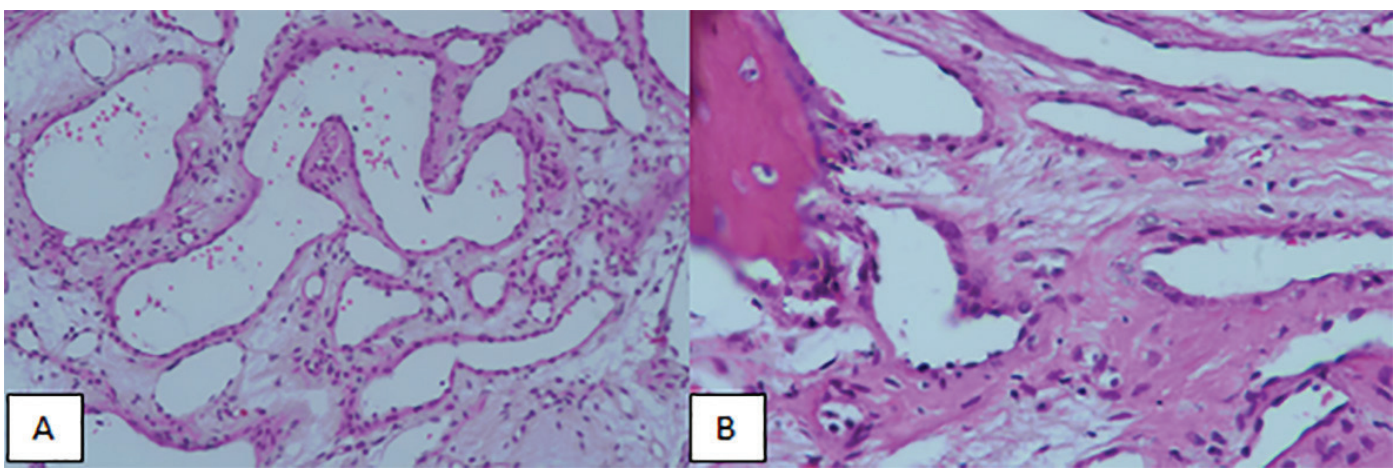

Figure 4: (A) Lesion consisting of thin-walled vascular structures (x200, H\&E). (B) Thin-walled vascular structures between bony trabeculae (x400, H\&E) H\&E: Hematoxylin and eosin

angiofibromas are other benign, locally aggressive hypervascular tumors of this region but are not associated with phleboliths and the predominant localization in the region of the sphenopalatine foramen and nasal cavity rather than the ITF. The permeative and destructive behavior of the lesion reminds a craniofacial sarcoma at first; however, the bright T2W signal is incompatible with a highly cellular sarcoma.

\section{CONCLUSION}

Venous VM should be considered in the differential diagnosis of skull base lesions, and phleboliths with bright T2W signals should be taken into account as distinguishing features.

\section{Ethics}

Informed Consent: Written informed consent has been taken from the patient's patents.

Peer-review: Externally peer-reviewed.

\section{Authorship Contributions}

Concept: B.E., T.K., E.K., S.A., C.C.., H.Ö., Design: B.E., T.K., E.K., N.K., Data Collection or Processing: O.I., B.E., Analysis or Interpretation: B.E., S.A., C.Ç., H.Ö., Literature Search: Ö.I., B.E., N.K., Writing: B.E.

Conflict of Interest: No conflict of interest was declared by the authors.

Financial Disclosure: The authors declared that this study received no financial support.

\section{REFERENCES}

1. Mulliken JB, Glowacki J. Hemangiomas and vascular malformations in infants and children: a classification based on endothelial characteristics. Plast Reconstr Surg 1982;69:412-22.
2. Lowe LH, Marchant TC, Rivard DC, Scherbel AJ. Vascular malformations: classification and terminology the radiologist needs to know. Semin Roentgenol 2012;47:106-17.

3. Resnick D. Vascular and lymphatic tumors. In: Draud LA, Fix CF, editors. Diagnosis of bone and joint disorders. 4th ed. Philadelphia, Pa: Saunders, 2002.4186-95.

4. Connor SE, Flis C, Langdon JD. Vascular masses of the head and neck. Clin Radiol 2005;60:856-68.

5. Buckmiller LM, Richter GT, Suen JY. Diagnosis and management of hemangiomas and vascular malformations of the head and neck. Oral Dis 2010;16:405-18.

6. Nagaoka M, Morino T. Venous malformation in the infratemporal fossa diagnosed by CT-guided core needle biopsy. BMJ Case Reports 2021;14:e238171.

7. Legiehn GM, Heran MK. Venous malformations: classification, development, diagnosis, and interventional radiologic management. Radiol Clin North Am 2008;46:545-97, vi.

8. Dubois J, Alison M. Vascular anomalies: what a radiologist needs to know. Pediatr Radiol 2010;40:895-905.

9. Hochman M, Adams DM, Reeves TD. Current knowledge and management of vascular anomalies, II: malformations. Arch Facial Plast Surg 2011;13:425-33.

10. Flors L, Leiva-Salinas C, Maged IM, Norton PT, Matsumoto AH, Angle JF, et al. MR imaging of soft-tissue vascular malformations: diagnosis, classification, and therapy follow-up. Radiographics 2011;31:1321-40; discussion 1340-1.

11. Dubois J, Soulez G, Oliva VL, Berthiaume MJ, Lapierre C, Therasse E. Soft-tissue venous malfor- mations in adult patients: imaging and therapeutic issues. Radio Graphics 2001;21:1519-31.

12. Navarro OM, Laffan EE, Ngan BY. Pediatric soft-tissue tumors and pseudo-tumors: MR imaging features with pathologic correlation: part 1. Imaging approach, pseudotumors, vascular lesions, and adipocytic tumors. Radiographics 2009;29:887-906. 